\title{
What is the Space of Spectral Sensitivity Functions for Digital Color Cameras?
}

\author{
Jun Jiang ${ }^{\dagger}$ \\ Dengyu $\operatorname{Liu}^{\dagger}$ \\ ${ }^{\dagger}$ Rochester Institute of Technology,
}

\author{
Jinwei $\mathrm{Gu}^{\dagger} \quad$ Sabine Süsstrunk ${ }^{\ddagger}$ \\ ${ }^{\ddagger}$ Ecole Polytechnique Fédérale de Lausanne
}

\begin{abstract}
Camera spectral sensitivity functions relate scene radiance with captured RGB triplets. They are important for many computer vision tasks that use color information, such as multispectral imaging, color rendering, and color constancy. In this paper, we aim to explore the space of spectral sensitivity functions for digital color cameras. After collecting a database of 28 cameras covering a variety of types, we find this space convex and two-dimensional. Based on this statistical model, we propose two methods to recover camera spectral sensitivities using regular reflective color targets (e.g., color checker) from a single image with and without knowing the illumination. We show the proposed model is more accurate and robust for estimating camera spectral sensitivities than other basis functions. We also show two applications for the recovery of camera spectral sensitivities - simulation of color rendering for cameras and computational color constancy.
\end{abstract}

\section{Introduction}

Camera spectral sensitivities are functions of wavelength describing the relative efficiency of light detection for color filters and image sensors. It relates scene radiance with recorded RGB values for a digital color camera. The knowledge of camera spectral sensitivities is important for many computer vision tasks that use color information, such as multispectral imaging [22, 17], color constancy [24, 3], and spectral reflectance recovery $[13,5]$.

Camera spectral sensitivities are often measured with a monochromator that generates narrow-band light and a spectrophotometer that measures its spectral power distribution [15]. This method, however, is applicable only in a laboratory setting, with a time-consuming scanning over the wavelength range of interest. Recent approaches simplify the recovery of camera spectral sensitivities by using specialized targets such as a fluorescent checker [6], a LED-based emissive chart [1], or multiple instead of a single picture of a color target [23]. Nevertheless, it is assumed $[1,7,8,19]$ that camera spectral sensitivities cannot be reliably recovered by using regular broadband reflective color targets (e.g., color checker) even with known illumi- nation. The reason is that real-world spectral reflectance has a lower intrinsic dimensionality than the number of unknowns of camera spectral sensitivities. The requirement of specialized devices or targets has become a hurdle for the estimation of camera spectral sensitivities, because compared to fluorescent or LED-based color targets, reflective color targets are still much more stable and easier to manufacture, maintain, and use.

On the other hand, for most digital color cameras, spectral sensitivity functions are designed with certain constraints in mind, which means they may reside in some low dimensional space. For instance, most digital color cameras are designed to make 'nice' pictures and the characteristics of the camera spectral sensitivities are a trade-off between maximizing quantum efficiency and minimizing noise.

Motivated by these observations, in this paper, we ask the question: what is the space of spectral sensitivity functions for digital color cameras? Finding a low-dimensional, statistical model for camera spectral sensitivities is useful for estimating them with fewer constraints. With this goal, our contributions in this paper are as follows:

- We have measured a database of spectral sensitivity functions for 28 cameras, including professional DSLRs, point-and-shoot, industrial and mobile cameras, as shown in Fig. 1. To our knowledge, this is the most extensive database of this kind so far. The database and source codes are available at http: //www.cis.rit. edu/jwgu.

- We perform principal component analysis (PCA) on this database and find that the space of camera spectral sensitivities is two dimensional.

- Using this PCA-based model, we propose two methods that estimate camera spectral sensitivities from a single image of the commonly used reflective color checker, for both known and unknown illumination.

- We also show several applications in color rendering after we estimate camera spectral sensitivities.

\section{Related Work}

Estimation of Camera Spectral Sensitivity Camera spectral sensitivities are usually measured using a monochromator and a spectrometer [15, 14], with a timeconsuming scanning over the wavelength range of interest. 


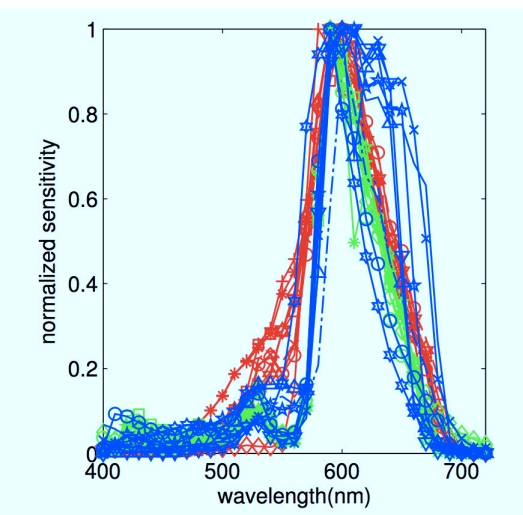

(a) Red channel

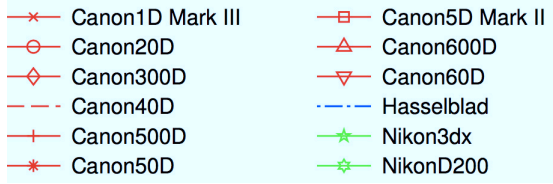

* Canon50D

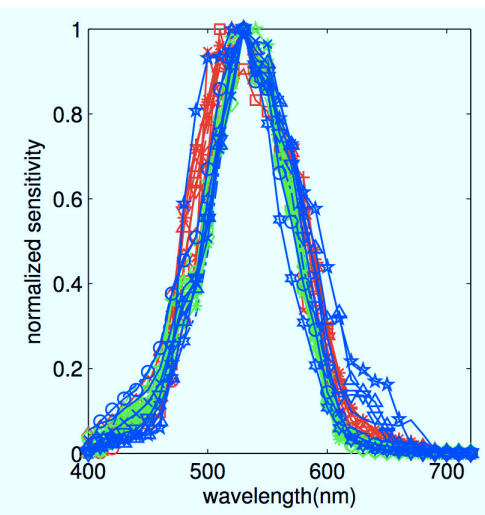

(b) Green channel

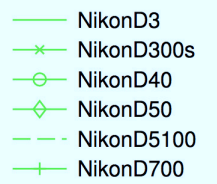

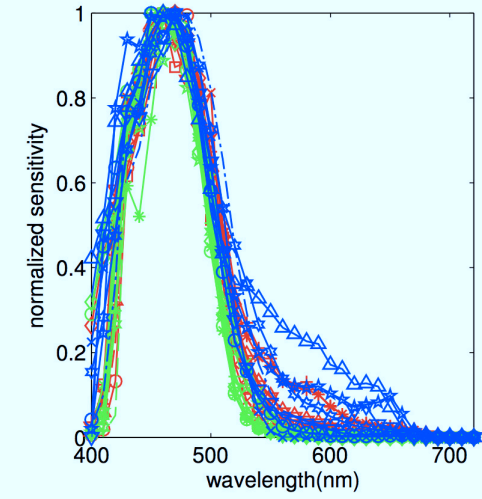

(c) Blue channel

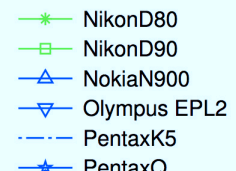

Phase One

- PointGreyGrasshopper

* PointGreyGrasshopper2

- - Sony Nex5N

Figure 1. The normalized spectral sensitivities of the 28 cameras in our database, including professional DSLRs, point-and-shoot, industrial and mobile cameras. Statistical analysis of these measurements shows the space of camera spectral sensitivities is two-dimensional. This statistical model is useful to recover camera spectral sensitivities from a single image with regular broadband reflective color targets.

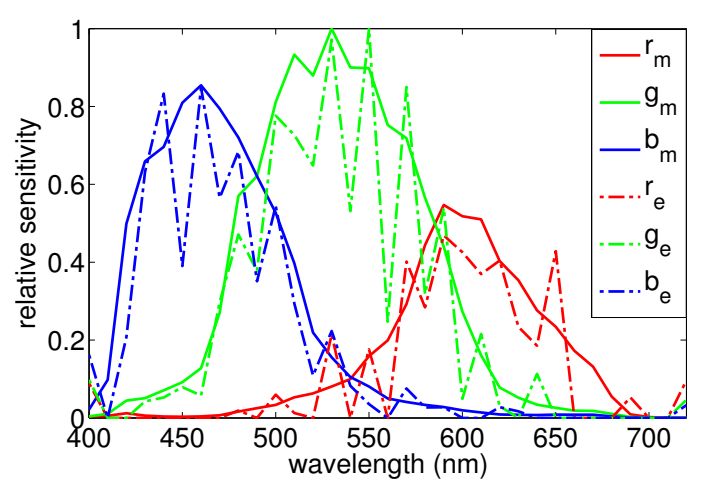

Figure 2. The need for statistics prior when estimating the camera spectral sensitivities. Direct inversion by Eq. (2) suffers even with a small amount of noise $(1 \%)$ due to the low dimensionality of spectral reflectance of real-world objects. The subscripts $m$ and $e$ stand for the measured and estimated camera spectral sensitivities.

This method is accurate but expensive. Hardeberg et al. [7] proposed to estimate the camera spectral sensitivities with known illumination and scene reflectance, but they found it unreliable due to the low intrinsic dimensionality of scene spectral reflectance $[9,7]$. To overcome this, Urban et al. [23] took multiple pictures of a color target under different LED illuminations. Dicarlo et al. [1] proposed to use a LED-based emissive target, and recently Han et al. [6] proposed to use a fluorescent color target.

\section{Statistical Analysis of Camera Response Functions} Our work is inspired by [4] that performed statistical analysis of camera radiometric response functions. Similar analysis has been performed for the spectra of daylight [10,21] and used for illumination and spectral reflectance recov- ery [16]. For camera spectral sensitivity functions, Zhao et al. [26] collected data for 12 cameras and compared four types of basis functions to model camera spectral sensitivities. They found the radial basis function optimal for recovering camera spectral sensitivities. In comparison, with more data, we find the PCA-based model more accurate. We also propose to recover spectral sensitivities from a single image under unknown illumination.

\section{The Space of Camera Spectral Sensitivity}

\subsection{Statistical Analysis of Spectral Sensitivity Func- tions for Digital Color Cameras}

We first introduce some background and show why it is necessary for statistical analysis of camera spectral sensitivities. The RGB triplet pixel intensities at one spatial position $x, I_{k, x}, k=R, G, B$, can be modeled as the product of the spectral reflectance of the point $R_{x}(\lambda)$, the spectral power distribution of the illuminant $L(\lambda)$, and the camera spectral sensitivities $C_{k}(\lambda), k=R, G, B$, integrating over the visible spectral range from $400 \mathrm{~nm}$ to $720 \mathrm{~nm}$,

$$
I_{k, x}=\int_{400 n m}^{720 n m} C_{k}(\lambda) L(\lambda) R_{x}(\lambda) d \lambda, \quad k=R, G, B .
$$

These equations can also be written in a matrix form, $\mathbf{i}_{\mathbf{k}}=$ $\mathbf{c}_{\mathbf{k}} \mathbf{L R}, k=R, G, B$, where $\mathbf{i}_{\mathbf{k}}$ is a $1 \times m$ vector $(m$ is the number of pixels), $\mathbf{c}_{\mathbf{k}}$ is a $1 \times 33$ vector (assuming we have 33 bands from $400 \mathrm{~nm}$ to $720 \mathrm{~nm}$ with an interval of $10 \mathrm{~nm}), \mathbf{L}=\operatorname{diag}(L(400 \mathrm{~nm}), \cdots, L(720 \mathrm{~nm}))$, and $\mathbf{R}=$ $\left[\mathbf{r}_{\mathbf{1}}, \cdots, \mathbf{r}_{\mathbf{m}}\right],\left(\mathbf{r}_{\mathbf{m}}=\left[R_{m}(400 n m), \cdots, R_{m}(720 n m)\right]^{T}\right)$.

To estimate camera spectral sensitivities $\mathbf{C}=$ $\left[\mathbf{c}_{\mathbf{R}}, \mathbf{c}_{\mathbf{G}}, \mathbf{c}_{\mathbf{B}}\right]^{T},(T$ is the transpose of the matrix), in the- 
ory we can take a picture of color patches with known reflectance, $\mathbf{R}$, under a known illuminant, and solve with pseudo-inverse

$$
\mathbf{c}_{\mathbf{k}}=\mathbf{i}_{\mathbf{k}} \cdot(\mathbf{L R})^{+}, k=R, G, B .
$$

In practice, however, as shown in Fig. 2, direct inversion cannot reliably recover camera spectral sensitivities. Even when we use 1269 Munsell color chips as the color target, direct inversion is still quite sensitive to noise. As pointed out in $[9,7]$, this is because spectral reflectance of realworld objects has a low intrinsic dimensionality (i.e., 6 or 8) [12, 18] which makes $\mathbf{R}$ rank deficient. To overcome the problem, recent work [1, 6] used either narrow-band LED illumination or fluorescent color targets. Nevertheless, it is highly desirable to solve this problem with reflective color targets, because they are easier to manufacture, maintain, and use. We show below that with statistical analysis of camera spectral sensitivity functions, we can find a low dimensional model of spectral sensitivities and solve this problem from a single image under unknown illumination.

\subsection{Assumptions and Constraints for Camera Spec- tral Sensitivity Functions}

In this paper, we are interested in the spectral sensitivity functions of digital color cameras in the visible spectrum (i.e., $400 \mathrm{~nm}$ to $720 \mathrm{~nm}$ ). To unify our discussion, we make the assumptions and constraints listed below.

- We assume the spectral sensitivity functions of a camera is spatially invariant, i.e., they are functions of wavelength only: $C_{k}(\lambda), k=R, G, B$.

- We also assume the spectral sensitivity functions are non-negative,

$$
C_{k}(\lambda) \geq 0, k=R, G, B .
$$

In addition, we normalize the spectral sensitivity function of each color channel to be between zero and one,

$$
\max _{\lambda} C_{k, n}(\lambda)=1, k=R, G, B,
$$

where $\mathbf{c}_{\mathbf{k}, \mathbf{n}}$ is the normalized spectral sensitivity, and $\mathbf{c}_{\mathbf{k}}=g_{k} \cdot \mathbf{c}_{\mathbf{k}, \mathbf{n}}, g_{k} \geq 0, k=R, G, B .{ }^{1}$ The absolute magnitudes of spectral sensitivities can be accounted by gain of an imaging system and thus can be excluded for modeling spectral sensitivities. Note that our normalization is different from previous work $[6,26]$ where only the peak of $C_{G}(\lambda)$ is normalized to one. We normalize such that the peak of all RGB channels to one, because we aim to use statistical models to explain the spread rather than the height variation of spectral sensitivity functions.

\footnotetext{
${ }^{1}$ After normalization, Eq. 1 becomes $I_{k, x}=\int_{400 \mathrm{~nm}}^{720 \mathrm{~nm}} g_{k}$. $C_{k, n}(\lambda) L(\lambda) R_{x}(\lambda) d \lambda, \quad k=R, G, B$. where $g_{k}$ is the constant for the red, green or blue channel, and $C_{k, n}(\lambda)$ is the normalized spectral sensitivity. The matrix form of Eq. 1 becomes $\mathbf{i}_{\mathbf{k}}=g_{k} \mathbf{c}_{\mathbf{k}, \mathbf{n}} \mathbf{L R}, \mathrm{k}=\mathrm{R}, \mathrm{G}$, $\mathrm{B}$, where $\mathbf{c}_{\mathbf{k}, \mathbf{n}}=\left[C_{k, n}(400 \mathrm{~nm}), \cdots, C_{k, n}(720 \mathrm{~nm})\right]$.
}

- To faithfully capture the color of a scene, in theory, cameras need to satisfy the Luther condition [15], i.e., the camera spectral sensitivity functions need to be a linear transformation of the CIE-1931 2-degree color matching functions:

$$
\left[\begin{array}{l}
\overline{\mathbf{x}} \\
\overline{\mathbf{y}} \\
\overline{\mathbf{z}}
\end{array}\right]=\mathbf{T}\left[\begin{array}{l}
\mathbf{c}_{\mathbf{R}} \\
\mathbf{c}_{\mathrm{G}} \\
\mathbf{c}_{\mathrm{B}}
\end{array}\right],
$$

where $[\overline{\mathbf{x}}, \overline{\mathbf{y}}, \overline{\mathbf{z}}]^{T}$ is the CIE-1931 2-degree color matching function, $\left[\mathbf{c}_{\mathbf{R}}, \mathbf{c}_{\mathbf{G}}, \mathbf{c}_{\mathbf{B}}\right]^{T}$ are the spectral sensitivities of a digital color camera, and $\mathbf{T}$ is a full-rank $3 \times 3$ matrix to be determined. In practice, however, due to limitations in hardware (e.g., color filters in the Bayer pattern), the Luther condition is often satisfied to a certain degree, especially for low-end consumergrade cameras.

Equations (3), (4), and (5) define the space of spectral sensitivity functions of digital color cameras. It is easy to see this space is a convex set. If $\mathbf{C}_{\mathbf{1}}$ and $\mathbf{C}_{\mathbf{2}}$ are in this set, any convex combination $a \mathbf{C}_{\mathbf{1}}+(1-a) \mathbf{C}_{\mathbf{2}}, 0 \leq a \leq 1$ must also be in this set. Below, we measured spectral sensitivity functions for a wide range of cameras and performed statistical analysis to chart the space.

\subsection{Database of Camera Spectral Sensitivity}

We have measured the spectral sensitivity functions for 28 cameras using a monochromator and a spectrometer PR655. The measurement setup and details can be found in the supplementary materials.

The raw measured data, after normalization, is in Fig. 1. Most spectral sensitivity functions peak at similar wavelength for each channel. To validate whether they satisfy the Luther condition, we estimate the matrix $\mathbf{T}$ with least square based on Eq. (5). The spectral Root Mean Square (RMS) error, $\left\|\mathbf{C}_{\mathbf{2 d e g}}-\mathbf{T} \cdot \mathbf{C}\right\|$, is used for evaluation, where $\mathbf{C}_{2 \text { deg }}$ is a matrix of the CIE-1931 2-degree color matching function, and $\mathbf{C}$ are the measured camera spectral sensitivities. Color difference (CIEDE00 [11]) is also calculated between $\mathbf{C}_{\mathbf{2} \text { deg }}$ and $\mathbf{T} \cdot \mathbf{C}$ under CIE D65 illuminant for the 1269 Munsell color chips [18]. Ideally, both the spectral RMS and color difference are zero if a camera perfectly satisfies Luther condition. As shown in Fig. 3, however, overall most cameras have a deviation from the Luther condition, especially for the two industrial cameras. It also shows that Canon cameras in general have lower RMS and color differences than Nikon cameras in this aspect.

\subsection{A PCA Model for Camera Spectral Sensitivity}

We performed Principal Component Analysis (PCA) on the normalized data for each color channel separately. In our database, we have 9 Canon and 10 Nikon cameras, and 


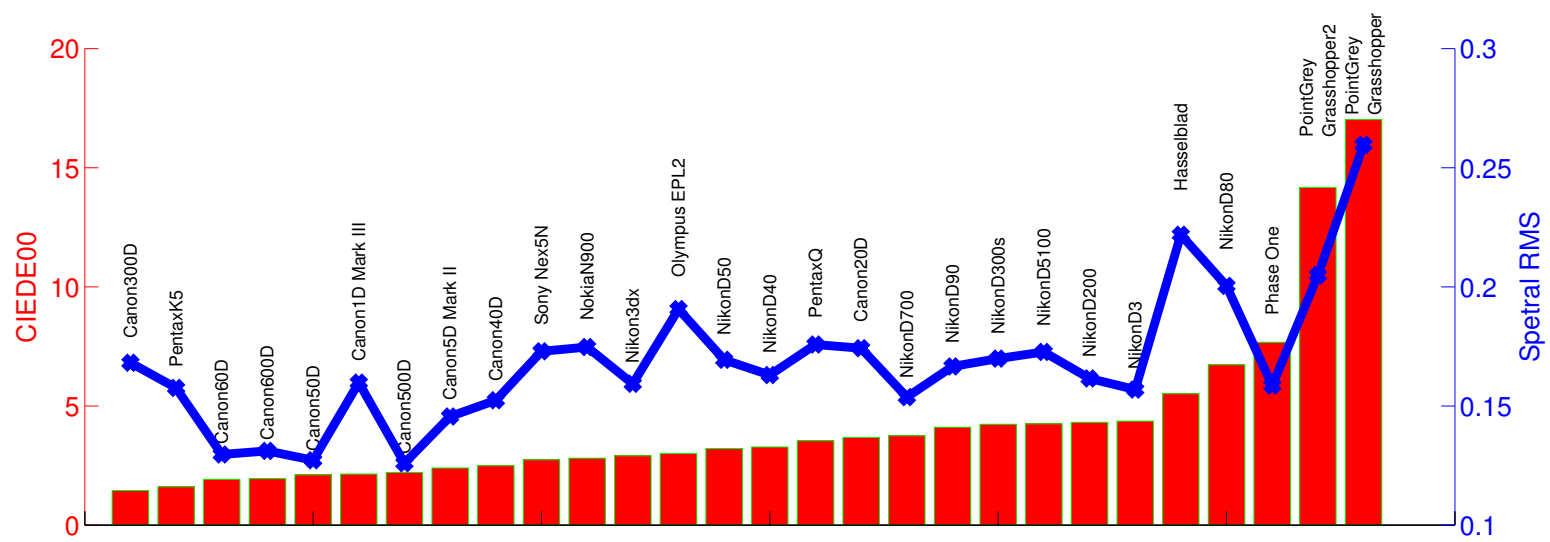

Figure 3. A camera satisfies the Luther condition if its spectral sensitivity function is a linear transformation of the CIE-1931 2-degree color matching function. The Luther condition can be evaluated by the RMS error between $\mathbf{C}_{\mathbf{2 d e g}}$ and $\mathbf{T} \cdot \mathbf{C}$, where $\mathbf{T}$ is computed by Eq. (5), $\mathbf{C}_{\mathbf{2} \text { deg }}$ are the CIE-1931 2-degree color matching functions, and $\mathbf{C}$ are the measured camera spectral sensitivities. Color difference (CIEDE00 [11]) is calculated between $\mathbf{C}_{\mathbf{2} \text { deg }}$ and $\mathbf{T} \cdot \mathbf{C}$ under CIE D65 illuminant and the 1269 Munsell color chips [18]. Ideally, spectral RMS and color differences are zero if a camera perfectly satisfies the Luther condition. Overall, most cameras have a deviation from the Luther condition, especially for the two industrial cameras.

9 other cameras. PCA was performed on all Canon cameras, all Nikon cameras, and all 28 cameras to investigate the difference across different types of cameras. The results are shown in Fig. 4. When all 28 cameras are used, the 1 st principal component explains over $95 \%$ of total variance of the data, and the first two principal components explain over $97 \%$, which means camera spectral sensitivities can be modeled as a two-dimensional space with reasonably high accuracy (which is also confirmed by our recovered results and applications). In other words, camera spectral sensitivities can be decomposed as $\mathbf{c}_{\mathbf{k}, \mathbf{n}}=\sigma_{\mathbf{k}} \cdot \mathbf{E}_{\mathbf{k}}, k=R, G, B$, where $\sigma=\left[\sigma_{1}, \sigma_{2}\right]$ is a $1 \times 2$ vector, $\mathbf{E}_{\mathbf{k}}=\left[\mathbf{e}_{\mathbf{k}, \mathbf{1}}, \mathbf{e}_{\mathbf{k}, \mathbf{2}}\right]^{T}$ is $2 \times 33$, the eigenvector matrix. The matrix form of Eq. 1 is

$$
\mathbf{i}_{\mathbf{k}}=g_{k} \sigma_{\mathbf{k}} \mathbf{E}_{\mathbf{k}} \mathbf{L R}, k=R, G, B
$$

where $\mathbf{E}_{\mathbf{k}}, k=R, G, B$ is shown in Fig. 4 , and the scatter plot of $\sigma$ of the 28 cameras is shown in Fig. 5.

\section{Spectral Sensitivity from a Single Image}

We show the PCA model enables the recovery of camera spectral sensitivities with commonly used reflective color targets from a single image.

\section{1. with a Known Light Source}

If the spectrum of a light source is known, we can capture an image of a reflective color target (e.g., color checker) and recover camera spectral sensitivities by $g_{k} \sigma_{\mathbf{k}}=$ $\mathbf{i}_{\mathbf{k}}\left(\mathbf{E}_{\mathbf{k}} \mathbf{L R}\right)^{+}, k=R, G, B$. The camera spectral sensitivities can be obtained by

$$
\mathbf{c}_{\mathbf{k}}=g_{k} \mathbf{c}_{\mathbf{k}, \mathbf{n}}=g_{k} \sigma_{\mathbf{k}} \mathbf{E}_{\mathbf{k}}=\mathbf{i}_{\mathbf{k}}\left(\mathbf{E}_{\mathbf{k}} \mathbf{L R}\right)^{+} \mathbf{E}_{\mathbf{k}}, k=R, G, B .
$$

\section{2. with an Unknown Light Source}

If the spectrum of a light is unknown but we know it is daylight, we show both the spectrum of daylight and the camera spectral sensitivities can be recovered, by using the daylight spectrum model [10]. This daylight model is represented as $L(\lambda, t)=\bar{L}(\lambda)+M_{1}(t) V_{1}(\lambda)+M_{2}(t) V_{2}(\lambda)$, where $M_{1}$ and $M_{2}$ are functions of correlated color temperature $(t), \bar{L}(\lambda)$ is the average daylight spectrum, and $V_{1}(\lambda)$ and $V_{2}(\lambda)$ are the characteristic vectors of daylight. In matrix form, the daylight model can be expressed as $\mathbf{l}(\mathbf{t})=\overline{\mathbf{l}}+M_{1}(t) \mathbf{v}_{\mathbf{1}}+M_{2}(t) \mathbf{v}_{\mathbf{2}}$ Thus, Eq. (7) becomes $\mathbf{C}_{\mathbf{k}}=\mathbf{i}_{\mathbf{k}}\left(\mathbf{E}_{\mathbf{k}} \mathbf{l}(\mathbf{t}) \mathbf{R}\right)^{+} \mathbf{E}_{\mathbf{k}}, k=R, G, B$. Both $\sigma$ and $t$ are optimized iteratively to minimize the RMS $\left\|\mathbf{i}_{\mathbf{k}}-g_{k} \sigma_{\mathbf{k}} \mathbf{E}_{\mathbf{k}} \mathbf{l}(\mathbf{t}) \mathbf{R}\right\|, k=R, G, B$.

Figure 6 shows an example where we use both methods to recover the spectral sensitivities of a Canon 60D camera. Figure 6(d) shows the result when the spectrum of daylight is known. Figures 6(e) and (f) show that without knowing the daylight, we can recover both the unknown daylight spectrum and the camera spectral sensitivities. The recovery of spectral sensitivities of more cameras can be found in the supplementary materials. We also evaluated this method under daylight at different time of the day in the supplementary material, which confirms the method stable.

\subsection{Comparison with Other Basis Functions}

We also compare this PCA model with three other basis functions for modeling camera spectral sensitivities [26], including Fourier, radial, and polynomial basis. Details of these basis functions are in the supplementary material.

Using the same captured image (Fig. 6(c)), we recover camera spectral sensitivities with the four types of basis functions, respectively. As shown in Fig. 7, the results are not as accurate as that by using the PCA model (Fig. 6(f)). We also use the recovered camera spectral sensitivities to simulate the rendering of a color checker under D65 illuminant. In Fig. 8, the PCA model outperforms other basis functions, resulting in the smallest color difference overall. 


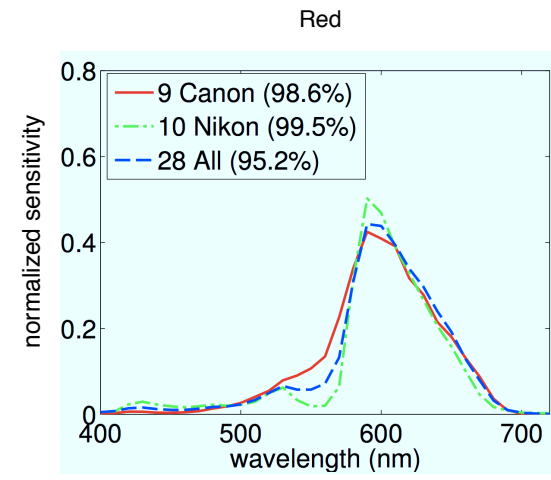

(a) 1st eigenvector $\left(e_{1}\right)$

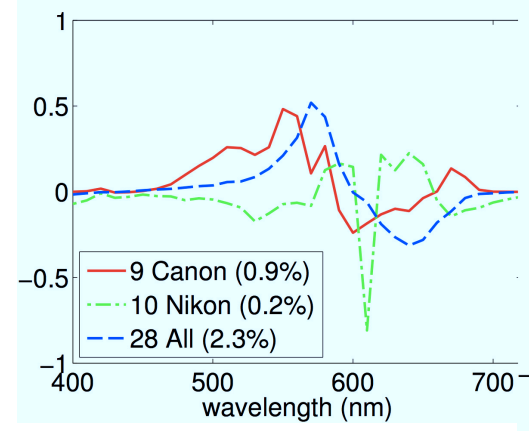

(d) 2nd eigenvector $\left(e_{2}\right)$

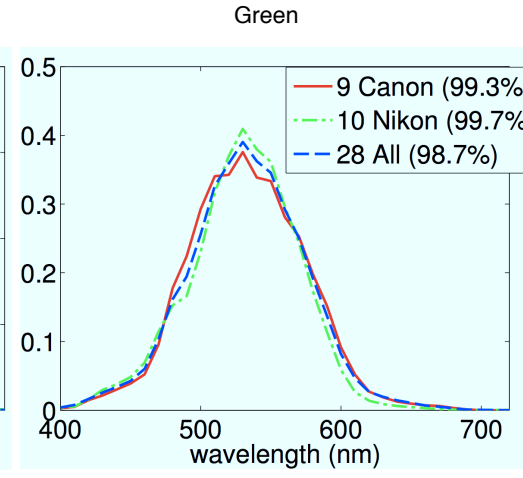

(b) 1st eigenvector $\left(e_{1}\right)$

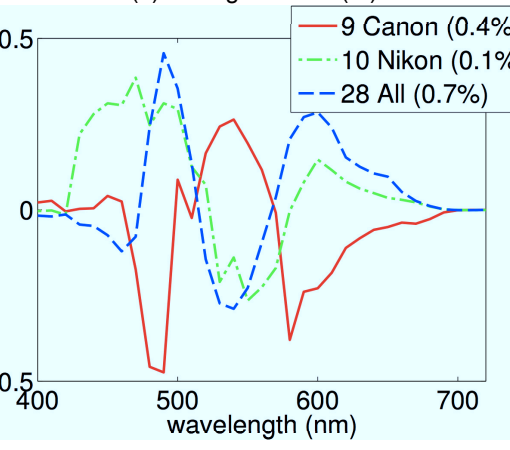

(e) 2nd eigenvector $\left(e_{2}\right)$
Blue

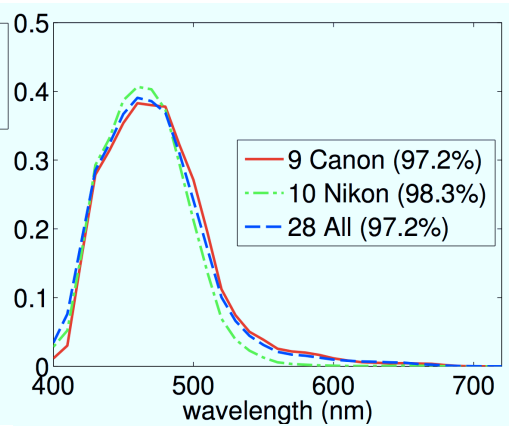

(c) 1st eigenvector $\left(e_{1}\right)$

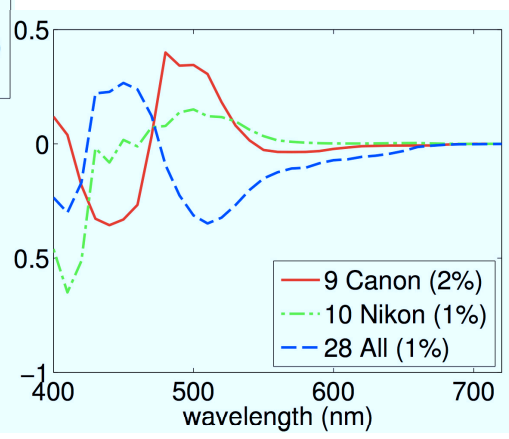

(f) 2nd eigenvector $\left(\mathrm{e}_{2}\right)$

Figure 4. The principal components of camera spectral sensitivities. Top Row: 1st principal component. Bottom Row: 2nd principal component. The three columns represent the R/G/B channels, respectively. We performed PCA on Canon cameras, Nikon cameras, and all 28 cameras. The 1st principal component accounts for over $95 \%$ of total variance for all three channels, and the first two principal components accounts for over $97 \%$ of total variance. Thus, we model camera spectral sensitivity functions as two-dimensional functions.

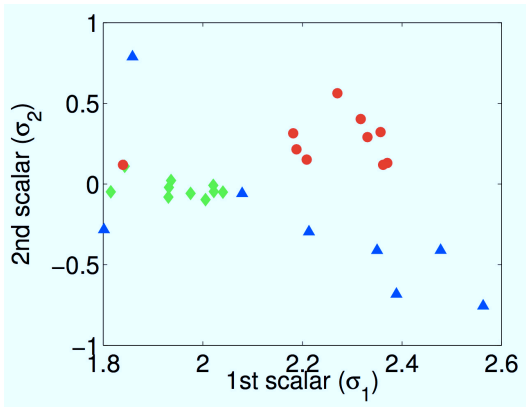

(a) Red

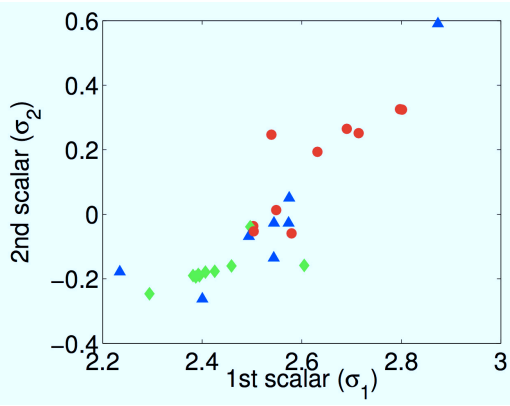

(b) Green

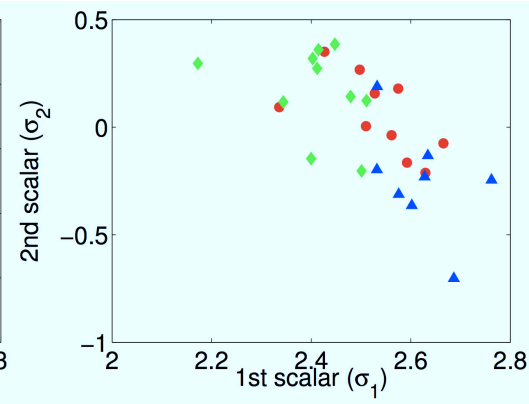

(c) Blue

Figure 5. The scatter plot of the 28 cameras in the two-dimensional space, for the red (a), green (b) and blue (c) channel. The red circles are Canon cameras, the green diamonds are Nikon cameras, and the blue triangles are the other cameras in the database.

We also evaluate the robustness to noise when recovering camera spectral sensitivities with the four types of basis functions over the entire database (i.e., 28 cameras). The proposed PCA model is more robust to noise, with the smallest RMS error in the recovery in Table 1, contradicting with that in [26]. We believe the reason is that our PCA model is derived with more data (i.e., 28 vs 12 cameras).

\section{Applications}

In this section, we show two applications after the recovery of camera spectral sensitivity functions.

\subsection{Simulation of Color Rendering for Cameras}

A straightforward application is to simulate the color rendering of a multi-spectral image for cameras, i.e., generating a RGB image for certain camera models. This is useful for the design and evaluation of cameras. Figure 9 shows an example where renderings are made using the measured and recovered camera spectral sensitivities of Canon 60D. The multispectral images are from the database [25]. Color difference is calculated, and they are close to one, indicating the accuracy of the recovered camera spectral sensitivities. 


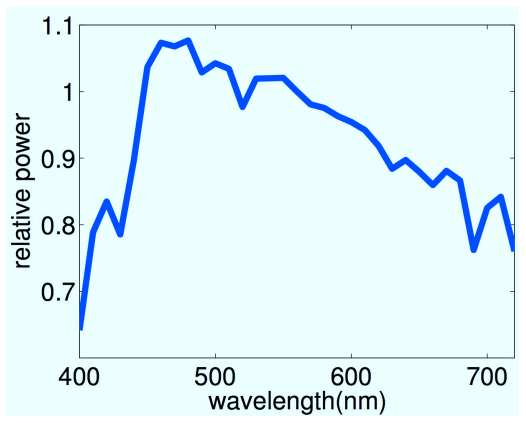

(a) Spectral power distribution of daylight

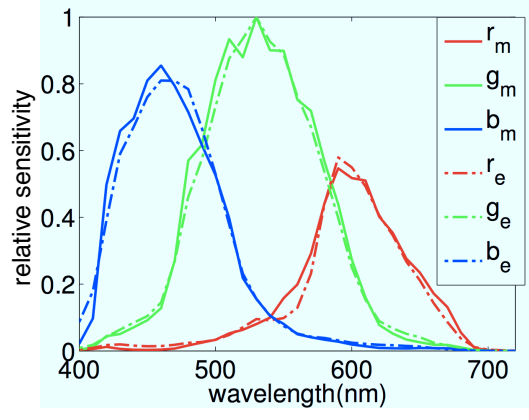

(d) Recovered camera spectral sensitivity with known daylight

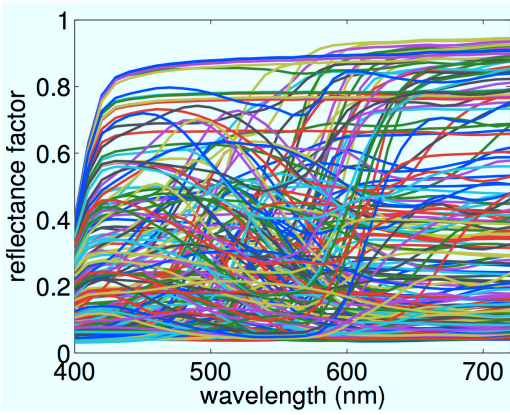

(b) Spectral reflectance of CCDC

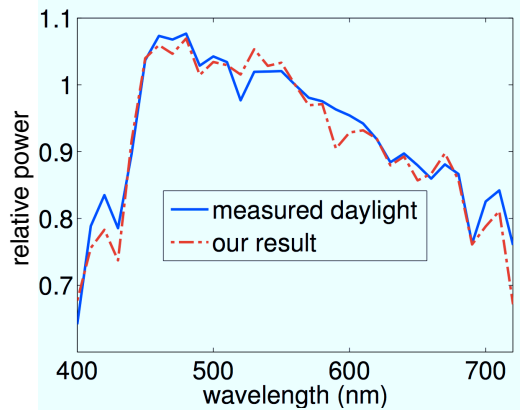

(e) Recovered daylight spectrum

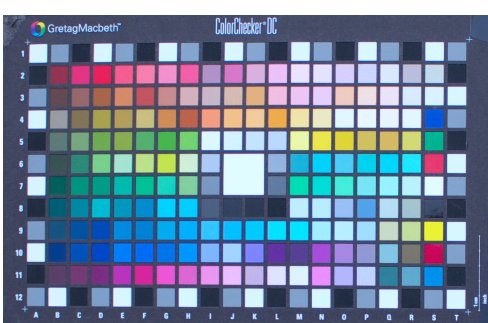

(c) The captured image

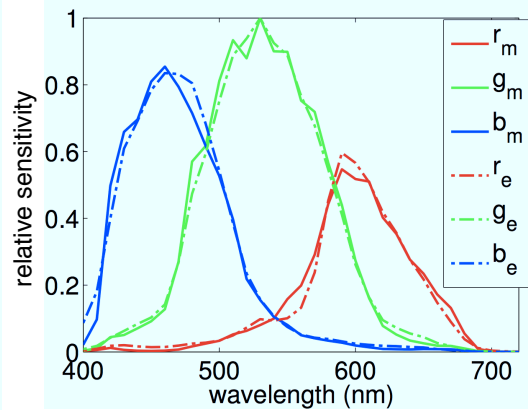

(f) Recovered camera spectral sensitivity with unknown daylight

Figure 6. The recovery of camera spectral sensitivities of Canon 60D. (a) The measured spectrum of a daylight. (b) The spectral reflectance of a color checker DC. (c) The captured image (glossy and duplicate patches are removed to avoid overweighting certain colors). (d) The recovered spectral sensitivities with known daylight spectrum. By using a daylight model, we can recover both the daylight spectrum (e) and the camera spectral sensitivities (f). The subscripts $m$ and $e_{e}$ in (d) and (f) stand for the measured and estimated camera spectral sensitivities, respectively.

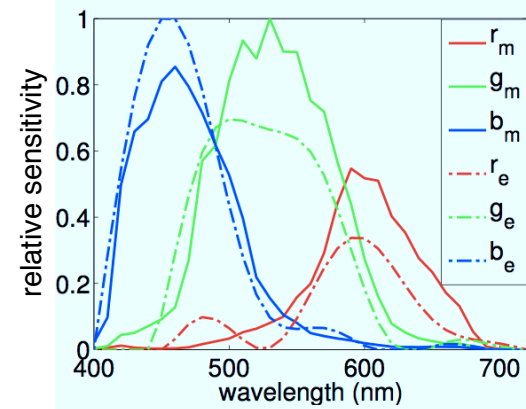

(a) Recovery by Fourier basis

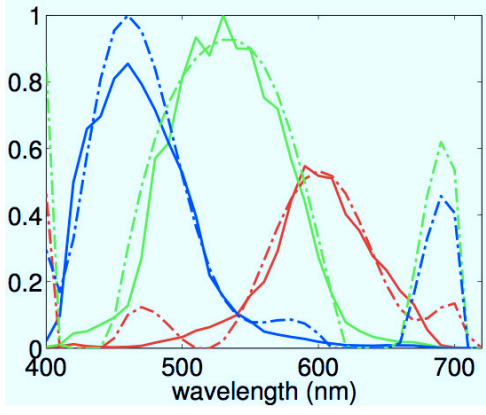

(b) Recovery by polynomial basis

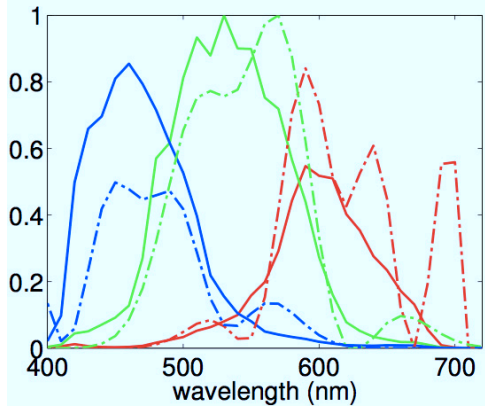

(c) Recovery by radial basis

Figure 7. The recovered camera spectral sensitivities of Canon 60D using other basis functions: (a) Fourier basis, (b) polynomial basis, and (c) radial basis. The results are worse than that of using the PCA model as shown in Fig. 6(f). The subscripts $m$ and $e$ stand for the measured and estimated camera spectral sensitivities, respectively.

\subsection{Computational Color Constancy}

Knowing camera spectral sensitivities is also useful for computational color constancy [2], i.e., removing the overall color cast in captured images. In order to recover the correct color of a scene, camera raw data needs to be converted to device-independent XYZ by Eq. (5), and then a chromatic adaptation transform is used to take care of the difference in the white point. A simple way to calculate the scene white point is $\left[\begin{array}{lll}X & Y\end{array}\right]_{w}^{T}=\mathbf{T} \cdot[R G B]_{w}^{T}$, where $[R G B]_{w}^{T}$ is the radiance of a white Lambertian area in the picture. A linear Bradford chromatic adaptation transform is used to convert to CIE D65, the white point of sRGB color space by default [2]. Computational color constancy relies on the accurate estimation of $\mathbf{T}$ (by Eq. (5)) and the white point of the scene. Knowing camera spectral sensitivities can help us estimate the matrix $\mathbf{T}$ correctly. 


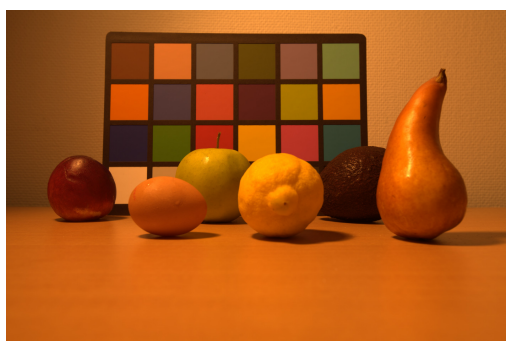

(a) Captured image

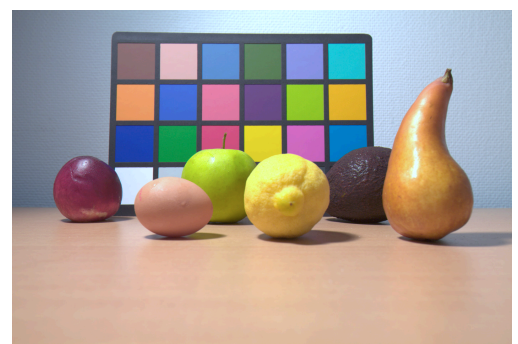

(b) Corrected image by knowing $T$

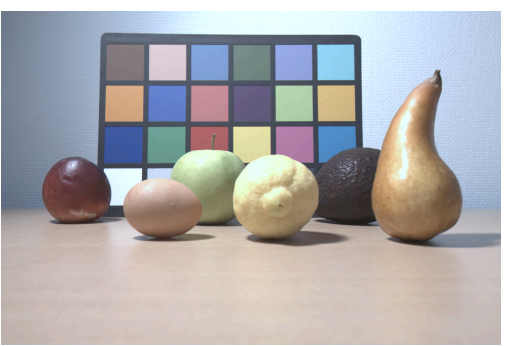

(c) Corrected image without knowing T

Figure 10. The correction of images by Canon 5D Mark II by removing the color cast in the image. A color checker is put in the scene for reference. The estimated camera spectral sensitivities of Canon 5D Mark II is used to calculate T by Eq. (5). (a) The captured image in sRGB. (b) The corrected image in sRGB based on $\mathbf{T}$ (c) The corrected image by dividing the white point (without using $\mathbf{T}$ ).

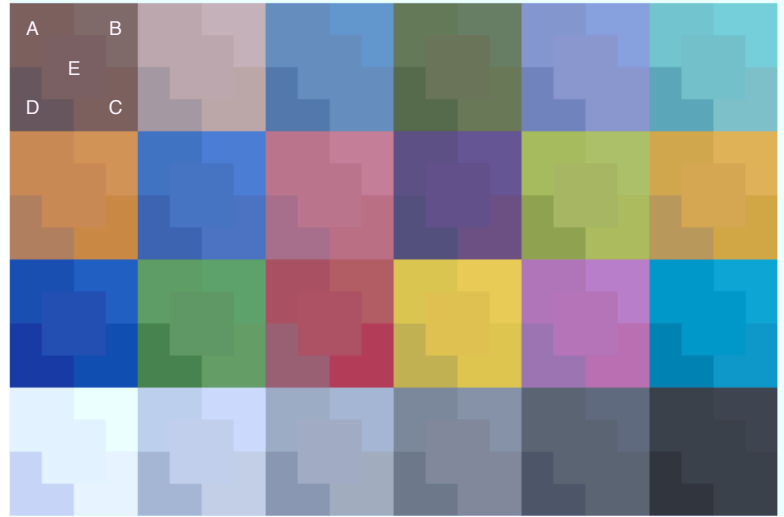

Figure 8. Comparison of four types of basis functions for modeling camera spectral sensitivity functions: A-PCA model (ours, Fig. 6(f)), B-Fourier basis (Fig. 7(a)), C-radial basis (Fig. 7(c)), and D-polynomial basis (Fig. 7(b)) with the ground truth (E). A color checker is rendered under D65 with camera spectral sensitivities recovered using these basis functions, and converted to sRGB. The average color difference between the renderings (from A to D) and the ground truth $(\mathrm{E})$ are 1.59, 3.54, 2.43 and 7 . The gain of the imaging system remains the same for all four basis functions. Table 1. The spectral RMS between the measured and recovered camera spectral sensitivities using four types of basis functions. For polynomial, Fourier, and radial, 8 basis functions are used [26]. At all noise levels, the PCA model outperforms other basis functions.

\begin{tabular}{|c|c|c|c|c|c|}
\hline \multirow{2}{*}{\multicolumn{2}{|c|}{ Spectral RMS }} & \multicolumn{4}{|c|}{ Basis functions } \\
\hline & & Eigenvector & Polynomial & Fourier & Radial \\
\hline \multirow{6}{*}{ 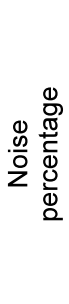 } & $0 \%$ & 0.057 & 0.131 & 0.091 & 0.13 \\
\hline & $1 \%$ & 0.058 & 0.133 & 0.091 & 0.131 \\
\hline & $6 \%$ & 0.059 & 0.145 & 0.104 & 0.155 \\
\hline & $10 \%$ & 0.058 & 0.163 & 0.121 & 0.193 \\
\hline & $47 \%$ & 0.072 & 0.279 & 0.197 & 0.289 \\
\hline & $78 \%$ & 0.106 & 0.327 & 0.264 & 0.317 \\
\hline
\end{tabular}

In Fig. 10, the picture was captured by Canon 5D Mark II, whose camera spectral sensitivities were estimated using a single picture under unknown daylight. $\mathbf{T}$ was obtained by Eq. (5) using the estimated camera spectral sensitivities. The corrected image (Fig. 10(b)) is based on the $\mathbf{T}$ matrix.
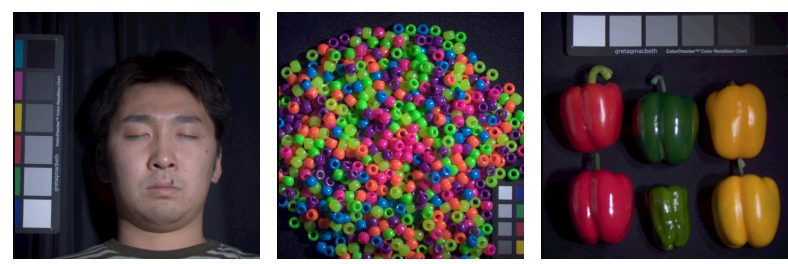

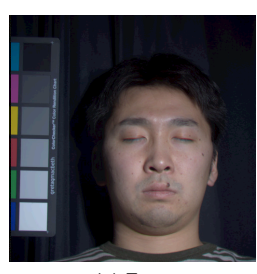

(a) Face

(CIEDE00: 1.34)

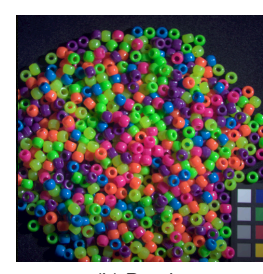

(b) Beads

(CIEDEO0: 0.9)

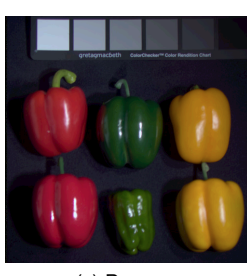

(c) Peppers (CIEDEOO: 1.18)
Figure 9. Simulation of color rendering for cameras. The images are rendered to sRGB based on the measured (top row) and estimated (bottom row) camera spectral sensitivities of Canon 60D. (a) face, (b) beads, and (c) peppers are from the multispectral image database [25]. The values in the parentheses are the average color difference (CIEDE00 [11]) between the bottom and top images in each column. For all three examples, the color difference is close to one, indicating a close color match.

Without camera spectral sensitivities, one of the methods to correct the images is to divide the white point RGB as shown in Fig. 10(c). By comparing the two corrected images, the color is more saturated and natural in Fig. 10(b) based on the recovered camera spectral sensitivities. More examples can be found in the supplementary material.

\section{Discussions}

In this paper, we explored the space of spectral sensitivity functions for digital color cameras. We measured a database of 28 cameras. We found the space of camera spectral sensitivities to be convex and two-dimensional. Based on the statistical model, we propose two methods to recover camera spectral sensitivities from a single image with and without knowing the illumination. Compared to other basis functions, we find the PCA-based model more accurate and robust to noise. We also showed several applications with the recovery of camera spectral sensitivities. 
The proposed method also has limitations. First, spurious measurements or outliers in the database may cause errors in the learned PCA model. Functional PCA [20] may be used to overcome this problem. Second, our method assumes we have access to camera raw data to ensure the linearity. When this assumption does not hold, both camera response function and camera spectral sensitivities have to be estimated simultaneously.

The proposed method works well under unknown daylight. Next, we are interested in the recovery of camera spectral sensitivities under general unknown illumination such as indoor overhead light or mixed lighting. Finally, we are also interested in applying the statistical model of camera spectral sensitivities to infer some spectral information for Internet photos.

\section{Acknowledgements}

This work was supported by NSF-1257163, NCCRMICS, Swiss National Science Foundation (5005-67322), a RIT OVPR grant, and a gift from Xerox. In addition, we also want to thank Prof. Susan Lakin in the School of Photography at RIT for providing the access to some cameras.

\section{References}

[1] J. M. Dicarlo, G. E. Montgomery, and S. W. Trovinger. Emissive chart for imager calibration. IS\&T/SID 12th Color Imaging Conference, pages 295-301, 2004. 4321, 4322, 4323

[2] M. D. Fairchild. Color Appearance Models. Wiley-IS\&T, 2005. 4326

[3] G. D. Finlayson and S. D. Hordley. Color constancy at a pixel. Journal of the Optical Society of America A, 18:253264, 2001. 4321

[4] M. D. Grossberg and S. K. Nayar. Modeling the space of camera response functions. IEEE Transactions on Pattern Analysis and Machine Intelligence, 26(10):1272-1282, 2004. 4322

[5] S. Han, I. Sato, T. Okabe, and Y. Sato. Fast spectral reflectance recovery using DLP projector. In Asian Conference on Computer Vision (ACCV), 2010. 4321

[6] S. Han, Y. Matsushita, I. Sato, T. Okabe, and Y. Sato. Camera spectral sensitivity estimation from a single image under unknown illumination by using fluorescence. In Proceedings of IEEE Conference on Computer Vision and Pattern Recognition (CVPR), 2012. 4321, 4322, 4323

[7] J. Y. Hardeberg, H. Brettel, and F. Schmitt. Spectral characterization of electronic cameras. Electronic Imaging: Processing, Printing, and Publishing in Color, 3409:100-109, 1998. 4321, 4322, 4323

[8] P. G. Herzog, D. Knipp, H. Stiebig, and F. Konig. Colorimetric characterization of novel multiplechannel sensors for imaging and metrology. Journal of Electronic Imaging, 8: 342-353, 1999. 4321

[9] P. M. Hubel, D. Sherman, and J. E. Farrell. A comparison of methods of sensor spectral sensitivity estimation. IS\&T/SID 2nd Color Imaging Conference, pages 45-48, 1994. 4322, 4323

[10] D. B. Judd. Spectral distribution of typical daylight as a function of correlated color temperature. Journal of Optical Society of America A, 54:1031-1040, 1964. 4322, 4324

[11] M. R. Luo, G. Cui, and B. Rigg. The development of the CIE 2000 colour-difference formula:CIEDE2000. Color Research and Application, 26:341-450, 2000. 4323, 4324, 4327

[12] L. T. Maloney. Evaluation of linear models of surface spectral reflectance with small numbers of parameters. Journal of Optical Society of America A, 3:1673-1683, 1986. 4323

[13] L. T. Maloney and B. A. Wandell. Color constancy: a method for recovering surface spectral reflectance. Journal of Optical Society of America A, 3:29-33, 1986. 4321

[14] C. Mauer. Measurement of the spectral response of digital cameras. VDM Verlag Dr. Mller, 2010. 4321

[15] J. Nakamura. Image Sensors and Signal Processing for Digital Still Cameras. CRC Press, 2006. 4321, 4323

[16] Y. Ohta and Y. Hayashi. Recovery of illuminant and surface colors from images based on the CIE daylight. In Proceedings of European Conference on Computer Vision (ECCV), volume 2, pages 235-246, 1994. 4322

[17] J. Park, M. Lee, M. D. Grossberg, and S. K. Nayar. Multispectral imaging using multiplexed illumination. In Proceedings of IEEE International Conference on Computer Vision (ICCV), 2007. 4321

[18] J. Parkkinen, J. Hallikainen, and T. Jaaskelainen. Characteristic spectra of Munsell colors. Journal of Optical Society of America A, 6(2):318-322, 1989. 4323, 4324

[19] S. Quan. Evaluation And Optimal Design of Spectral Sensitivities for Digital Color Imaging. $\mathrm{PhD}$ thesis, Rochester Institute of Technology, 2002. 4321

[20] J. O. Ramsay and B. W. Silverman. Functional Data Analysis. Springer-Verlag, New York, 1997. 4328

[21] D. Slater and G. Healey. What is the spectral dimensionality of illumination functions in outdoor scenes? In Proceedings of IEEE Conference on Computer Vision and Pattern Recognition (CVPR), 1998. 4322

[22] S. Tominaga. Spectral imaging by a multichannel camera. Journal of Electronic Imaging, 8:332-341, 1999. 4321

[23] P. Urban, M. Desch, K. Happel, and D. Spiehl. Recovering camera sensitivities using target-based reflectances captured under multiple LED-illuminations. 16th Workshop on Color Image Processing, pages 9-16, 2010. 4321, 4322

[24] B. A. Wandell. The synthesis and analysis of color image. IEEE Transactions on Pattern Analysis and Machine Intelligence, 9:2-13, 1987. 4321

[25] F. Yasuma, T. Mitsunaga, D. Iso, and S. Nayar. Generalized Assorted Pixel Camera: Post-Capture Control of Resolution, Dynamic Range and Spectrum. Technical Report CUCS061-08, Columbia University, Nov 2008. 4325, 4327

[26] H. Zhao, R. Kawakami, R. T. Tan, and K. Ikeuchi. Estimating basis functions for spectral sensitivity of digital cameras. In Meeting on Image Recognition and Understanding (MIRU), 2009. 4322, 4323, 4324, 4325, 4327 


\section{Supplementary Materials What is the Space of Spectral Sensitivity Functions for Digital Color Cameras?}

\author{
Jun Jiang ${ }^{\dagger} \quad$ Dengyu Liu ${ }^{\dagger}$ \\ $\dagger$ Rochester Institute of Technology,
}

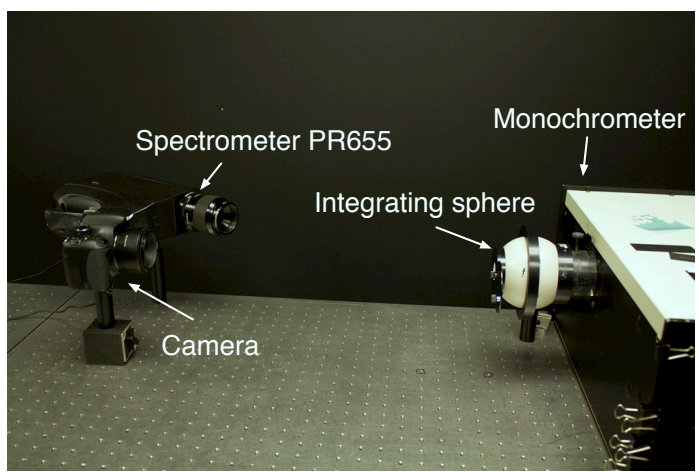

Figure 1. Experimental setup to obtain the ground truth of camera spectral sensitivity.

\section{Experimental Setup to Measure the Ground Truth of Camera Spectral Sensitivity}

As shown in Fig. 1, we have measured the spectral sensitivity functions for 28 cameras, including professional DSLRs, point-and-shoot, industrial and mobile cameras (i.e.Nokia N900), using a monochromator and a spectrometer PR655. At each wavelength, the camera spectral sensitivity in RGB channels is calculated by $c(\lambda)=$ $d(\lambda) /(r(\lambda) \cdot t(\lambda))$, where $d$ is the raw data recorded by the camera, $r$ is the illuminant radiance measured by the spectrometer, and $t$ is the exposure time of the camera. All other settings (i.e., ISO and aperture) remained the same during the measurement for each camera. The procedure is repeated across the whole visible wavelength from 400 to $720 \mathrm{~nm}$ with an interval of $10 \mathrm{~nm}$.

\section{Recovery of Camera Spectral Sensitivity Us- ing Other Basis Functions}

To fully evaluate the recovery performance using eigenvectors extracted from camera spectral sensitivities, we compared the recovery by using other basis functions. Zhao et al. [2] tested three basis functions besides camera space eigenvectors, and they are polynomial, Fourier, and radial basis functions. Zhao et al. [2] concluded that radial basis functions are the best.

\author{
Jinwei $\mathrm{Gu}^{\dagger} \quad$ Sabine Süsstrunk ${ }^{\ddagger}$ \\ ${ }^{\ddagger}$ Ecole Polytechnique Fédérale de Lausanne
}

The equation for the basis functions can be found here [2]. However, for completeness, these equations are listed in the paper. The equation for the Fourier basis function is expressed as

$$
\mathbf{F}=\sum_{i=0}^{D} a_{i} \cdot \sin (i \lambda \pi),
$$

where $\lambda$ is the wavelength vector normalized to be between 0 and 1. The Fourier basis functions are shown in Fig. 2(a).

The polynomial basis function is expressed as

$$
\mathbf{F}=\sum_{i=0}^{D} a_{i} \cdot \lambda^{i}
$$

where $\lambda$ is the wavelength vector from $400 \mathrm{~nm}$ to $720 \mathrm{~nm}$ with an interval of $10 \mathrm{~nm}$. It is normalized to be between 0 and 1 . The recovered spectral sensitivity, $\mathbf{F}$ is a linear combination of $\lambda^{i}$. The polynomial basis functions are shown in Fig. 2(b).

The radial basis functions are expressed as

$$
\mathbf{F}=\sum_{i=0}^{D} a_{i} \cdot \exp \left(-\frac{\left(\lambda-\mu_{i}\right)^{2}}{\sigma^{2}}\right),
$$

where $\lambda$ is the wavelength vector normalized to be between 0 and $1 . \mu_{i}$ and $\sigma^{2}$ are the peak wavelength and the variance of each basis function. The radial functions are shown in Fig. 3(a), (b) and (c) for the red, green, and blue channels. Eight basis functions are selected for the polynomial, Fourier, and radial method [2].

\section{Robustness of Spectral Sensitivity Recovery to Daylight Variation}

Judd [1] proposed that the daylight spectrum can be well represented using only a few parameters. To fully evaluate our recovery of camera spectral sensitivity under daylight, we simulated radiance using daylight measured at different time of the day, based on which the camera spectral sensitivity is recovered. The measured and recovered camera spectral sensitivity was then compared and spectral RMS calculated. The mean RMS for all 28 cameras in the database 


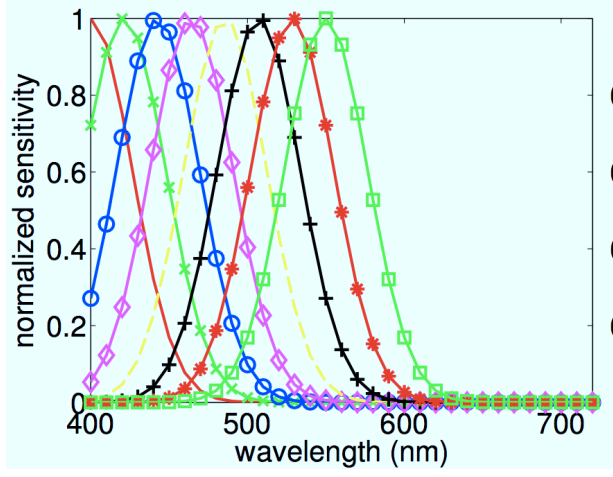

(a) Radial basis (blue)

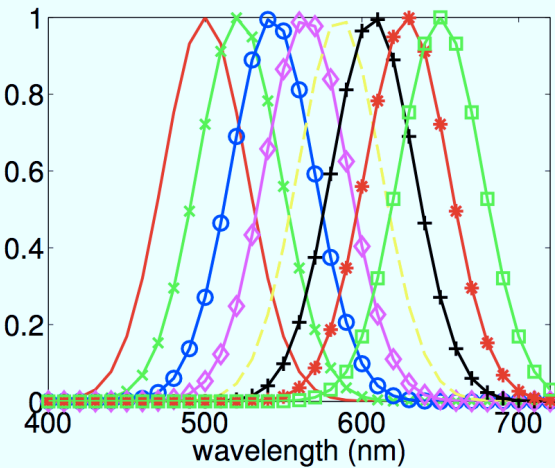

(b) Radial basis (green)

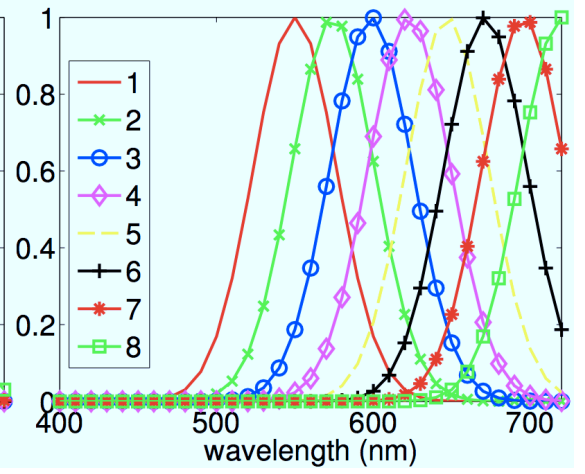

(c) Radial basis (red)

Figure 3. The radial basis functions of the (a) red, (b) green, and (c) blue channel.

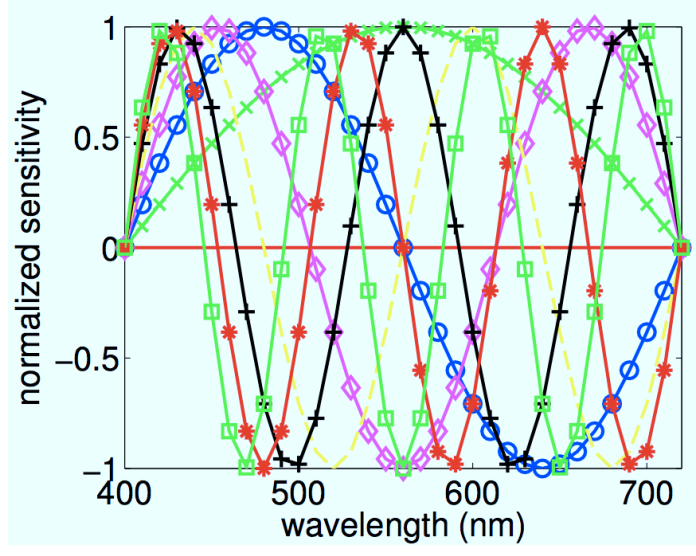

(a) Fourier basis

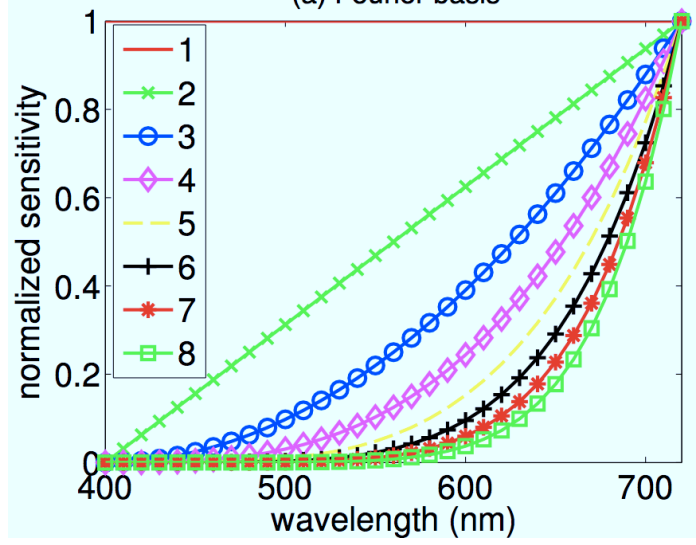

(b) Polynomial basis

Figure 2. The Fourier basis and polynomial basis functions.

is in Fig. 4. The recovery accuracy is about 0.06 , almost invariant to daylight at different time of the day.

\section{Dimensionality of Spectral Sensitivity}

While the camera spectral sensitivity is of high dimension (i.e.33 if the wavelength ranges from $400 \mathrm{~nm}$ to $720 \mathrm{~nm}$

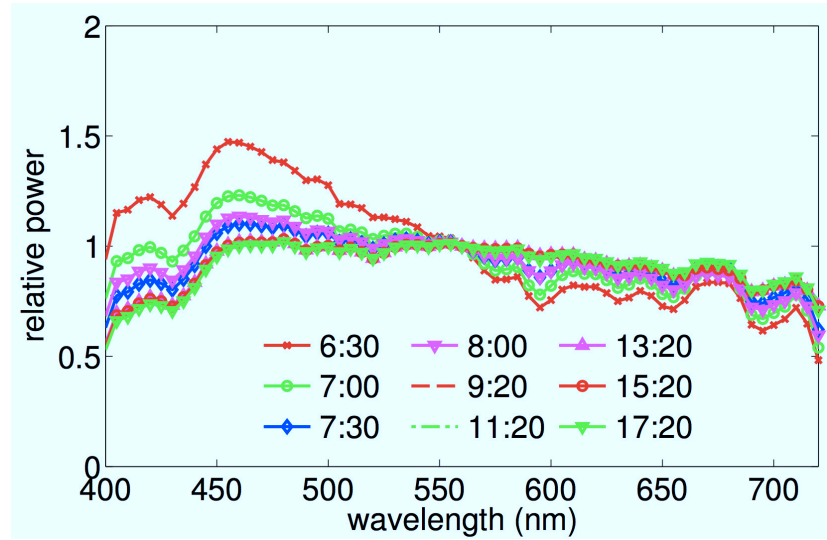

(a) Daylight measured at different time of the day

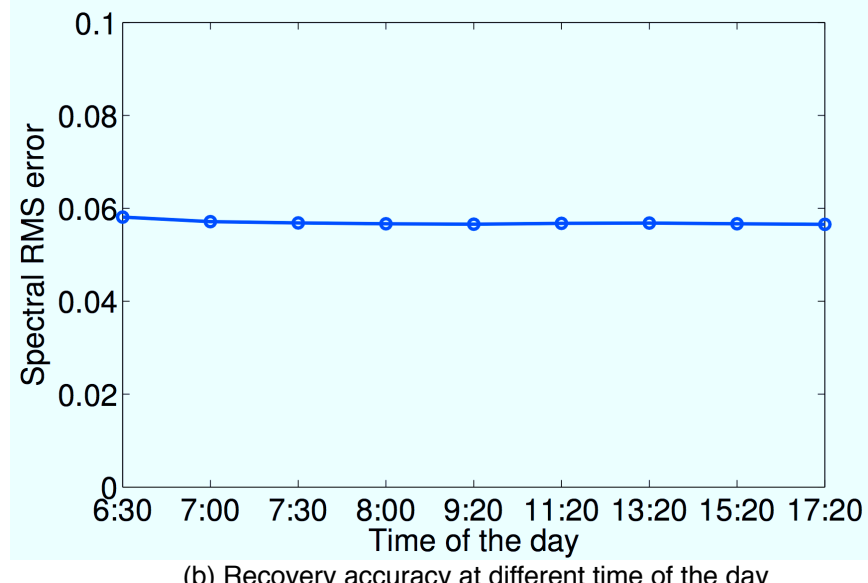

Figure 4. The spectral RMS error between the recovered and measured camera spectral sensitivity at different time of the day.

with an interval of 10nm), it can be represented using much fewer parameters. The variance that can be explained given the number of eigenvectors retained in the model is shown in Fig. 5. With two eigenvectors, we found that the camera spectral sensitivity can be well represented. 


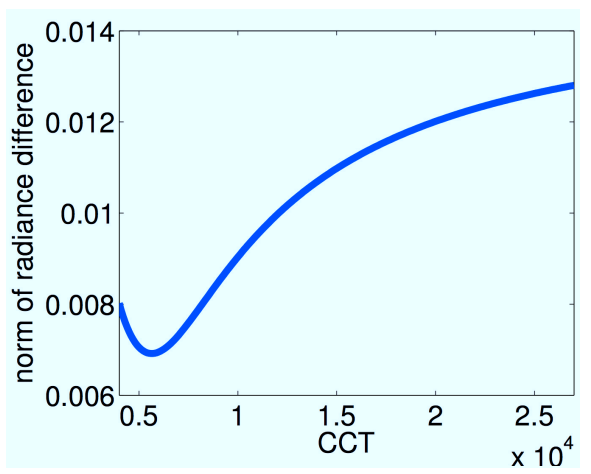

(a) Radiance error v.s. CCT

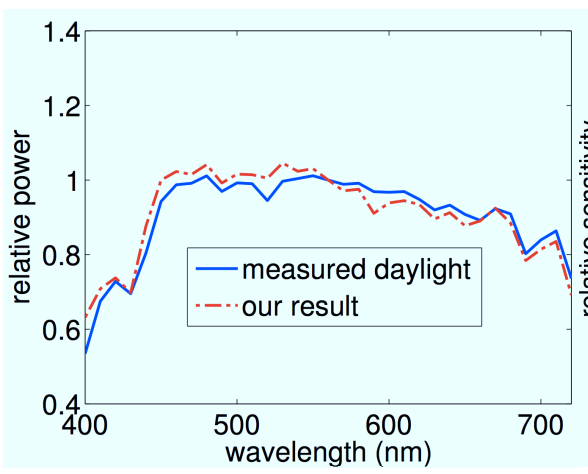

(b) Recovered daylight spectrum

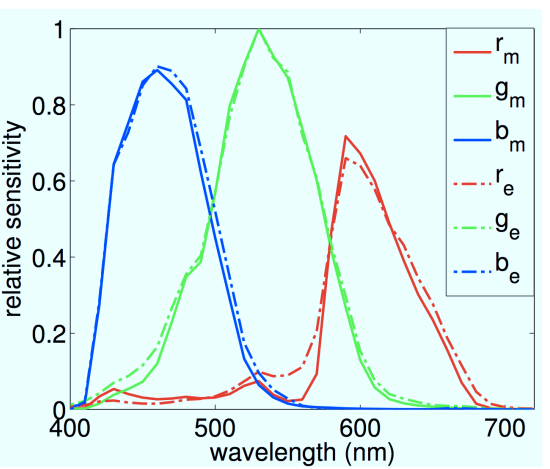

(c) Recovered camera spectral sensitivity with unknown daylight

Figure 6. The recovery of the camera spectral sensitivity of NikonD3 using a single picture of CCDC under unknown daylight. (a) The radiance error given the estimated camera spectral sensitivity at a certain CCT. The daylight spectrum that yields the lowest radiance difference is plotted in (b) and compared with the ground truth. (c) The measured and recovered camera spectral sensitivity of NikonD3. The subscripts $m$ and $e$ stand for the measured and estimated camera spectral sensitivity.

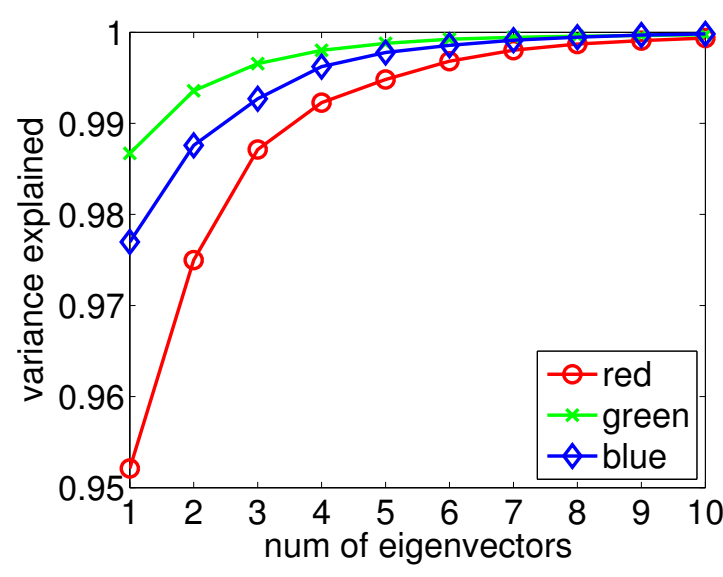

Figure 5. The percentage of total variance of the camera spectral sensitivity explained given the number of eigenvectors retained in the model. The first two eigenvectors are found to be enough to represent the space of camera spectral sensitivity.

\section{Results on Spectral Sensitivity Recovery}

We recovered the camera spectral sensitivity of NikonD3 using a picture of CCDC under unknown daylight. The radiance error given the CCT of daylight is in Fig. 6(a). The daylight spectrum that yields the least radiance eror is selected, and it is plotted in Fig. 6(b) with the measured daylight spectrum. A close match can be found between our recovered daylight and the ground truth. The recovered and measured camera spectral sensitivity are shown in Fig. 6(c). Similarly, the camera spectral sensitivity of a smartphone camera, NokiaN900, and another DSLR, Canon5D Mark II are recovered in Fig. ??.

\section{Results on Computational Color Constancy}

Accurate color corrections of images can be made by knowing the camera spectral sensitivity. In order to recover the correct color of a scene, camera raw data needs to be converted to device-independent XYZ by Eq. (5) in the paper, and then a chromatic adaptation transform (i.e.a linear Bradford transform) is used to take care of the difference in the white point. Computational color constancy relies on the accurate estimation of $\mathbf{T}$ (by Eq. (5)) and the white point of the scene. Knowing camera spectral sensitivity can help estimate $\mathbf{T}$ correctly. Examples are shown in Fig. 8. The color cast in the captured images in Fig. 8 is removed successfully by knowing $\mathbf{T}$ estimated from the recovered camera spectral sensitivity of Canon 5D MarkII. On the other hand, the corrected images are less saturated by dividing the white point (without knowing the $\mathbf{T}$ matrix).

\section{References}

[1] Deaane B. Judd. Spectral distribution of typical daylight as a function of correlated color temperature. Journal of the Optical Society of America, 54:1031-1040, 1964. 1

[2] Hongxun Zhao, Rei Kawakami, Robby T. Tan, and Katsushi Ikeuchi. Estimating basis functions for spectral sensitivity of digital cameras. Meeting on Image Recognition and Understanding 2009, 2009. 1 

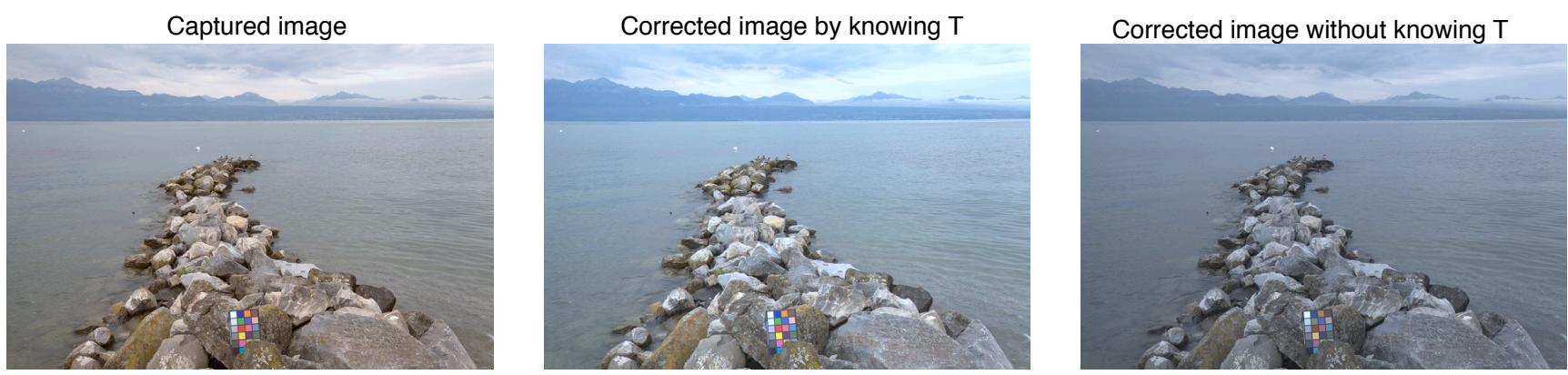

(a) Lake
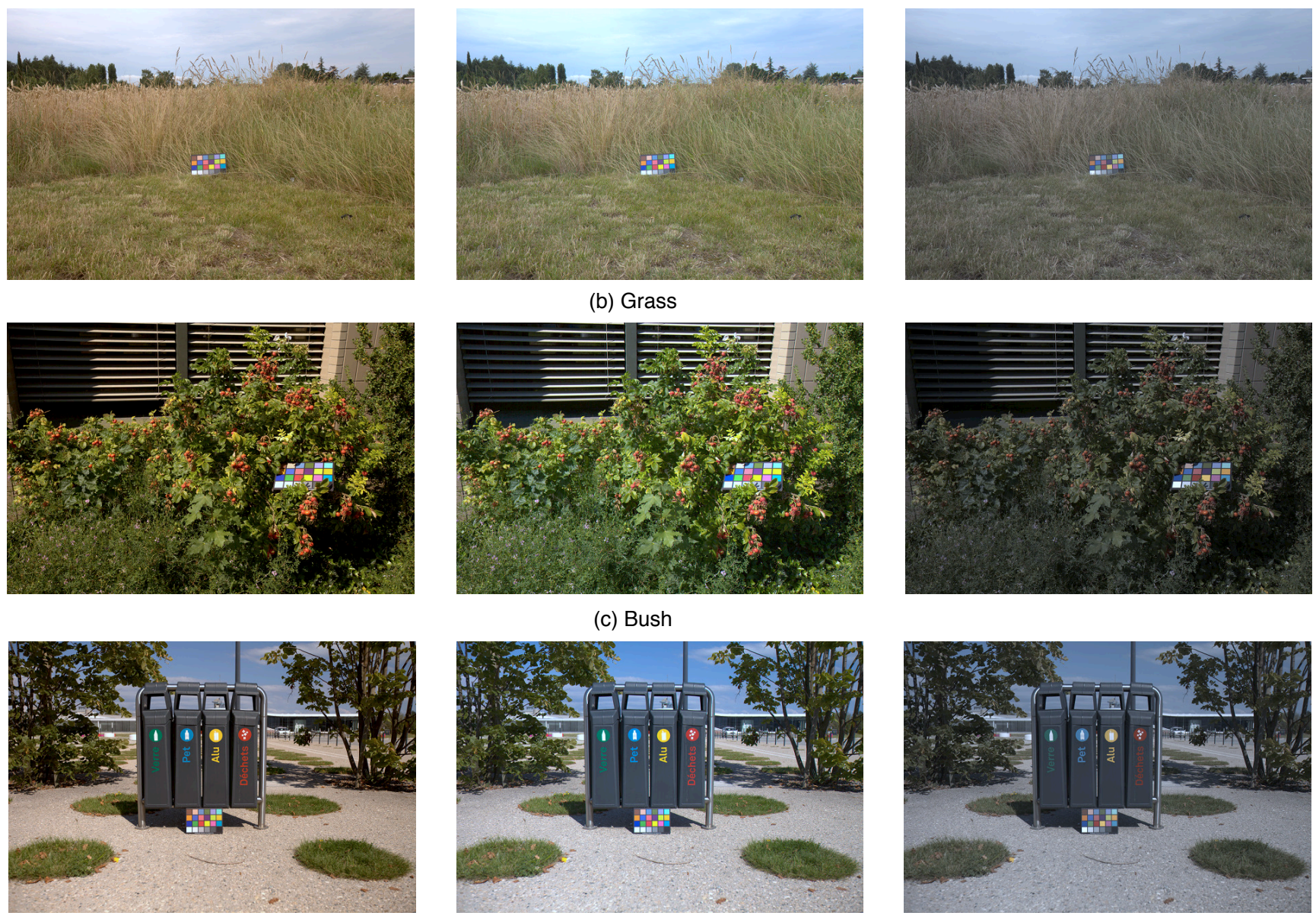

(d) Trash cans

Figure 8 . The correction of images by Canon5D Mark II by removing the color cast in the image. CC is put in the scene to locate the white point. The estimated camera spectral sensitivity of Canon5D Mark II is used to calculate $\mathbf{T}$ by Eq. (5). Left column: The captured image; Middle column: the corrected image based on $\mathbf{T}$, and Right column: the corrected image by dividing the white point (without using $\mathbf{T}$ ). The images are rendered in SRGB color space. 